Jorge L. Tizón

\title{
Bases para un equipo de atención precoz a los pacientes con psicosis
}

RESUMEN: Pródromos, la duración de la fase prodrómica y los factores de riesgo en la infancia y adolescencia de los cuadros psicóticos y, en especial, del «desequilibrio psicótico postpuberal» o «esquizofrenia».

PALABRAS CLAVE: Psicosis incipiente, pródromos de psicosis, esquizofrenia.
SUMMARY: Prodromic symptoms, the duration of the prodromic phase and the risk factors in the childhood and adolescence of the psychotic disorders and, especially, of the «postpuberal psychotic disorder» or schizophrenia.

KEY WORDS: Early psychosis, prodroms of psychosis, schizophrenia.

\section{Introducción}

Los «síndromes esquizofrénicos» y, en general, los trastornos psicóticos son los trastornos mentales que, probablemente, suponen más sufrimientos y costos tanto psicológicos (individuales y familiares) como micro y macrosociales (tabla 1). En las actuales condiciones de asistencia, el primer contacto con los servicios de SM de los pacientes diagnosticados de «psicosis esquizofrénica» y, en general, en todas las psicosis «delirantes»o «funcionales», usualmente está precipitado por los dramáticos síntomas y trastornos psicosociales del primer episodio (1-9). En la mayoría de los casos, el evento se halla precedido por una acumulación más o menos rápida de síntomas no específicos, síntomas «en negativo»y, finalmente, síntomas «en positivo», como ya describieron los primeros estudiosos del síndrome esquizofrénico (Bleuler, Kraepelin, Conrad, etc.).

¿Cuál es la experiencia personal, como sujetos, de los pacientes diagnosticados de «esquizofrenia» o «psicosis»? ¿Cómo viven la aparición de los primeros malestares, alteraciones, síntomas, tanto ellos como sus familias? Desde el punto de vista asistencial y técnico, esas tremendas experiencias, con las frustraciones, limitaciones y sufrimientos que conllevan, ¿pueden ayudarnos en la prevención, el tratamiento, la atención precoz? O, por el contrario, ¿esas vivencias y comunicaciones son sólo «aconteceres humanos» que no poseen ningún valor para la ciencia y la técnica, «excrecencias» y «actos vacíos de sentido», «discursos sin sentido para la ciencia y para la técnica?». Y si poseen sentido y valor para las aproximaciones asistenciales y para el estudio científico de tales formas del sufrimiento humano (10), ¿cómo usarlos en nuestras aproximaciones y técnicas de cuidado? ¿Con qué actitud, con qué instrumentos, con qué ética, con qué epistemología? Por ejemplo, si los estudiamos, ¿nos servirán para detectar precozmente esos trastornos psicopatológicos de las relaciones humanas y para ayudar 
a esos pacientes y grupos humanos desde los primeros síntomas, desde los pródromos? Más allá aún: ¿existen factores de riesgo detectables en la infancia y adolescencia que puedan avisarnos del posible desarrollo de una esquizofrenia o de una psicosis en la edad adulta? Y si existen ¿se puede incidir en ellos desde su manifestación en los dispositivos de Atención Primaria de Salud y en la interfase salud mental-atención primaria de salud?

Una vez que el trastorno está declarado, ¿cómo viven en nuestras sociedades los pacientes psicóticos que no ingresan, y sus familias? ¿Visitan los dispositivos de salud mental comunitaria, los médicos de familia o más bien tienden a perderse «en los intersticios de la sociedad y de sus propios repliegues psíquicos»? ¿Qué magnitudes suponen sus diversos subgrupos asistenciales: 1) los que ingresan a menudo; 2) los que no lo hacen pero están en contacto con los servicios de salud mental extrahospitalarios; 3) los que sólo conectan con los médicos de familia y enfermeras, con la APS, 4) los que desaparecen de las redes asistenciales durante años, hasta la próxima crisis aguda? (9-26).

Häffner y otros (1) han resumido los trabajos internacionales (Alemania, Suecia, USA, Australia, etc.) en los que se ha estudiado la duración del trastorno antes del primer ingreso o del primer contacto terapéutico (4-6). A pesar de las dificultades metodológicas de varios de tales estudios y de su disparidad tanto cultural como metodológica y de enfoque, casi todos ellos muestran que pasan varios años entre los primeros síntomas prodrómicos y el primer tratamiento, es decir, que el período de psicosis no tratada dura varios años en nuestras sociedades. En ese sentido, la vieja discusión entre «episodio»y «brote» (sin relación con la vida anterior del individuo) no es sostenible hoy. Se sabe cada vez más de los síntomas prodrómicos de la esquizofrenia, es decir, del amplio período que pasa entre sus primeras manifestaciones y el reconocimiento y primeros tratamientos del trastorno, la DUP, Duration of Untreated Psychoses, de los anglosajones (1; 8; 15-16; 27-30). Pero es que, además, tanto las investigaciones retrospectivas como las prospectivas aportan datos que hacen pensar en la existencia de síntomas neurológicos menores y, desde luego, en la existencia de numerosas diferencias entre el desarrollo de los niños y adolescentes que luego van a ser esquizofrénicos y otros sujetos de control $(8 ; 20 ; 31-36)$.

Por otra parte, en la mayoría de los casos, el primer episodio manifiesto de la psicosis se halla precedido por una acumulación de síntomas no específicos, síntomas «en negativo»y, finalmente, síntomas «en positivo». Es la acumulación de síntomas y perturbaciones a lo largo de los meses y los años o, más bien, la «ruptura del self» que los provoca, lo que hace aparecer en el ámbito social los «síntomas en positivo», usualmente los primeros que suelen valorarse como «trastorno». El paciente suele acabar entonces ingresado o, en otros casos, comienza su trata- 
ORIGINALES Y REVISIONES

miento sin necesidad de ingresar, etc. Usualmente, tendemos a pensar que ese es el «primer episodio» de la psicosis, que ingreso y «primer episodio» son eventos coincidentes. Empero, como apuntamos (10), en realidad ese paciente suele llevar ya varios «episodios» agudos o subagudos de sufrimiento y con síntomas: es la deformación tecnocrática y hospitalocéntrica de nuestros conocimientos lo que nos hace pensar que el trastorno no existe hasta que el paciente y/o su familia contactan con nosotros, con los «técnicos», con la «red profesional de asistencia».

Para los que trabajamos durante decenios en contacto clínico con esos pacientes y sus allegados, una primera preocupación es aclarar cómo conviven los pacientes psicóticos y sus familias con un trastorno tan grave y doloroso. En especial, cómo y desde cuándo comenzó el trastorno y cuáles fueron sus percepciones, cogniciones conflictos y sentimientos entonces. La hipótesis que hace decenios va calando en la psiquiatría mundial es que, si esos antecedentes se pudieran detectar con suficiente validez y fiabilidad, se podrían tratar antes y mejor $(1 ; 6 ; 8 ; 11$; $15 ; 28 ; 30 ; 37-48)$. El grupo Australiano y otros $(7-8 ; 49)$ han resumido los trabajos internacionales (Alemania, UK, Suecia, Finlandia, USA, Australia, etc.) en los que se ha estudiado la duración y avatares del trastorno antes del primer ingreso o del primer contacto terapéutico.

Yendo más atrás en esa retrospección-prospección, todas las hipótesis etiológicas actuales sobre las psicosis obligan a pensar en factores de riesgo y vulnerabilidades desde la infancia en la mayoría de casos y cuadros (46-47; 50-58). La literatura sobre factores de riesgo en la infancia y la adolescencia ha crecido exponencialmente, pero aún no existe un amplio acuerdo internacional sobre la importancia relativa de dichos factores de riesgo y, en especial, sobre las formas y métodos para localizarlos suficientemente fiables (18; 59-62). Por ejemplo, tanto las investigaciones retrospectivas como prospectivas cada vez aportan más datos que hacen pensar en la existencia de síntomas conductuales, cognitivos, emocionales y neurológicos menores, así como en la existencia de numerosas diferencias entre el desarrollo de los niños y adolescentes que luego van a ser catalogados como «esquizofrénicos»y otros sujetos de control, incluso hermanos de los afectados $(7-8 ; 18 ; 53 ; 59)(7-8 ; 18 ; 20 ; 31-36 ; 53 ; 59)$. Hay investigaciones que apuntan incluso a la existencia de diferencias visibles desde el primer año de la vida $(13 ; 19 ; 35 ; 63-66)$.

Uno de los problemas no resueltos consiste en que muchos de tales datos a menudo se asientan en estudios que metodológicamente poseen importantes lagunas y, por otro lado, son poco específicos. En realidad, en la mejor de las posibilidades, predicen trastornos mentales en los casos detectados, sin diferenciar demasiado si los mismos aparecerán en la propia infancia, en la adolescencia o en la edad adulta, ni sobre los diagnósticos con los que dichos individuos tenderán a 
ser diagnosticados. Esas dificultades metodológicas resultan comprensibles si tenemos en cuenta la complejidad de realizar un estudio prospectivo que necesitaría seguir poblaciones de 10.000 personas o 3.000 pacientes psiquiátricos para detectar nueve casos al año, complejidad que aumenta con los problemas éticos y económicos de ese tipo de estudios $(19 ; 35 ; 67-69)$.

Tal vez una vía para salir del bloqueo teórico y metodológico de tales estudios consistiría en intentar buscar, más que factores de riesgo específicos, «constelaciones» $\mathrm{o}$ «agrupaciones» de los mismos en las diversas edades de la infancia y la adolescencia dentro de los antecedentes de dichos pacientes. Todo ello sin olvidar que el concepto de «esquizofrenia» e incluso el de «psicosis» está necesitado de profundos replanteamientos tanto teóricos como epistemológicos, sin los cuales la investigación e incluso la clínica se hallan abocadas a frecuentes callejones sin salida. Y si avanzamos hacia la pubertad y la adolescencia, ha de tenerse en cuenta datos hoy confirmados como los del estudio $\mathrm{ABC}$ alemán, replicado entre nosotros en Cantabria -y que estamos intentando desarrollar en nuevos campos y poblaciones-. Los europeos (cf. 19; $24 ; 28 ; 70-71$ ) tienden a presentar el primer signo del trastorno a los 22, 5 años y las europeas a los 25,4 años. Pero el $73 \%$ de los mismos experimentaron una larga fase prodrómica de una media de 5 años con síntomas psicóticos ya manifiestos, pero sin diagnóstico ni tratamiento. Los estudios alemanes, así como los australianos, norteamericanos, suecos, fineses, etc., han descrito asimismo el tipo y características de esos primeros síntomas.

Hace años que investigamos sobre esos temas, en especial, dentro del Proyecto SASPE y el Proyecto LISMEN (19-20; 60). Uno de los objetivos era realizar una primera aproximación a esos síntomas y signos prodrómicos, así como a los factores de riesgo en la infancia y adolescencia. El equipo investigador proponía, en primer lugar, recoger datos sobre todos los pacientes que padecen esquizofrenia y psicosis en dos barriadas determinadas de la conurbación de Barcelona. ¿Cuántos son esos pacientes? (Tablas 2 y 3). Los datos provenientes de encuestas poblacionales con estos tipos de trastornos dan numerosos «falsos negativos»y «falsos positivos». De ahí que interesara una cuantificación real, porque, según nuestros datos, la mayoría de estos pacientes graves no ingresan en hospitales o lo hacen entre 2 y 4 veces en toda su vida. Cada año, de nuestra muestra de 476 pacientes esquizofrénicos y 362 con «otras psicosis», ingresan en servicios hospitalarios especializados únicamente el $8 \%$, con gran tendencia a los reingresos entre los que ingresan más de una ocasión (hay un número de pacientes que nunca ingresan, otros que lo hacen entre 1 y 4 ocasiones y otros que sufren numerosos reingresos). Hemos podido determinar tasas de incidencia y prevalencia en población general y en población de riesgo comprobadas para la población estudiada, así como otra serie de características 
ORIGINALES Y REVISIONES

psico-sociales: edad de aparición, distribución por géneros, barriadas y grupos sociales, fertilidad y fecundidad de los sujetos, etc. También algunos datos iniciales acerca de la influencia de los factores sociales en la diferenciación de la psicopatología y la eclosión y evolución de los trastornos. En una de nuestras muestras de esquizofrénicos que se visitaron durante un año concreto (2004), utilizando como herramienta la entrevista semiestructurada IRAOS-ERIE (Interview for the Retrospective Assessment of the Onset of Schizophrenia) (1), pudimos determinar que los síntomas prodrómicos más frecuentes en esa submuestra eran, por este orden: a) Las alteraciones de conducta (conducta extravagante, desorganizada y peleas); b) Ideación delirante (percepción megalomaníaca, ideas paranoides y delirio de referencia); c) El retraimiento o la euforia; d) Los problemas relacionales (de pareja o familiar, laborales y sexuales); e) Las alteraciones del sueño. Unos resultados algo diferentes de los publicados en los estudios australianos (8). Por otra parte, en esta submuestra del estudio SASPE hemos encontrado síntomas psicopatológicos definidos ya 5 años antes del primer episodio (20). En la mayoría de los casos, esos síntomas aparecen entre 1 y 5 años antes del primer episodio que se trata. En una minoría de los casos, hasta 10 años antes del diagnóstico. La moda en esta primera muestra (N = 22) se sitúa en los 3 años: son datos sobre la DUP (Duration of Untreated Psychoses) en estos pacientes.

Ahora bien, ¿existen medios para mejorar la detección y, por lo tanto, para acortar la duración del período sin tratamiento de la psicosis? El interés de los mismos resulta obvio: si los síntomas se pudieran detectar antes, el trastorno y sus pródromos se podrían atender antes. Con el efecto sobreañadido de que algunos estudios muestran ya que un tratamiento psicosocial precoz mejora la evolución de dichos pacientes (y disminuye el sufrimiento de sus familias) $(7 ; 45 ; 48 ; 72-73$ ). Es decir que, con una menor DPUP (Duration of Psychosocially Untreated Psychoses) la evolución tiende a cambiar en sentido positivo (8; 74-77). Resulta crucial la determinación tanto de los factores de riesgo como de los factores protectores de la aparición del primer episodio y de las recaídas. Usualmente se atribuye un papel fundamental a la red sanitaria entre esos factores de protección, pero ello implica el apriorismo de pensar que la mayoría de tales pacientes visitan los dispositivos de salud durante el año, algo que está por demostrar, incluso en nuestras sociedades tecnológicas, y que nuestros datos provisionales se contradicen. En realidad, sabemos poco de cómo viven en nuestras sociedades los pacientes con psicosis y sus familias cuando no ingresan. Ni siquiera estamos suficientemente seguros de la magnitud que suponen esos diversos subgrupos asistenciales que antes hemos citado: a) los que ingresan con elevada frecuencia; b) los que no ingresan pero están en contacto con los servicios de salud mental extrahospitalarios; c) los que sólo conectan con los profesionales de la Atención 
Primaria de Salud; d) los que se evaden de las redes asistenciales durante años, y sólo reaparecen en las crisis agudas $(10 ; 18 ; 67 ; 78)$. Pero incluso cuando los síntomas prodrómicos ya están definidos (cuando el trastorno psicótico ya sería evidente si el paciente consultase), en el caso de los sujetos que no ingresen en la red sanitaria, tampoco sabemos para ese período cuáles son las «vías» o «caminos» que siguen esos pacientes y sus familias para evitar o «rodear» la asistencia y para encontrar una nueva posición social en la que ubicarse. Anteriormente, las investigaciones sociológicas, y hoy las estrictamente epidemiológicas y psiquiátricas, han proporcionado abundantes datos acerca de cómo ese nuevo lugar social implica importantes pérdidas de salud en la familia, pérdidas en su calidad de vida, ruptura de lazos con la red social, etc. $(23-24 ; 48 ; 68 ; 78)$. Consecuentemente, tiende a pensarse que una mayor DUP (Duration of Untreated Psychoses) se acompaña de mayores pérdidas y disfunciones familiares, sociales y personales y de severas pérdidas en la calidad de vida. Sin embargo, hay que aclarar que hoy por hoy se trata de una hipótesis que cuenta con datos a favor pero que aún no podemos dar como comprobada $(17 ; 76)$ y que debe ser replanteada desde la perspectiva de las «terapias integradas», es decir, que utilizan tratamientos psicológicos y psicosociales en la atención precoz. Por eso antes hablaba de la diferenciación entre DUP (Duration of Untreated Psychoses) y DPUP (Duration of Psychosocially Untreated Psychoses) o DPTPP (Duración del Período sin tratamiento psicosocial de la Psicosis).

Lo que resulta claro, desde luego, es que las características tanto de los pródromos como de las vías de acceso o evitación de los tratamientos están sujetas a numerosas influencias, tanto psicosociales (individuales y familiares) como sociales: organización del sistema sanitario y el sistema social, ideología y cultura de la población, importancia de la estigmatización, tipo de dispositivos existentes, accesibilidad de los diversos tipos de tratamientos, cultura de grupo y esperanza institucional de los dispositivos funcionantes, etc. $(19 ; 70-71 ; 79)$. Por otra parte, tampoco está clara la definición del «síndrome prodrómico» y su diferenciación con la «psicosis no tratada» ni poseemos medios suficientemente válidos para definir a esos sujetos en pródromos, trema, «estado mental de alto riesgo»o «psicosis incipiente» (40).

Ahora bien, la clínica de los equipos de atención precoz de al menos tres continentes apunta cotidianamente a la importancia del tratamiento precoz de los niños, púberes y adolescentes que presentan muchos de esos tipos de déficits funcionales y relacionales. Y muestra también, cada vez con mayor frecuencia, importantes resultados positivos tanto de numerosas intervenciones precoces en la infancia $(58 ; 80)$ como del tratamiento precoz de las psicosis y los pródromos de psicosis $(7-8 ; 58 ; 65-66 ; 81-82)$. Los resultados preventivos se observan cuando los tratamientos no son meramente farmacológicos y, cuando, además de realizar 
ORIGINALES Y REVISIONES

el necesario apoyo psicológico del niño, púber o adolescente, se centran en el apoyo y contención psicosocial de la familia y los núcleos vivenciales naturales de desarrollo del niño $(58 ; 66 ; 83)$. Es decir, cuando se trata de una auténtica «terapia combinada» 0 «integrada» $(84 ; 48)$. Los equipos escandinavos hablan del «tratamiento adaptado a las necesidades», pero preferimos hablar del «tratamiento adaptado a las necesidades del paciente y su familia dentro de la comunidad» (TAN-PFC).

Todo eso hace pensar en la necesidad de instaurar procedimientos y equipos para la detección y atención precoz de los pacientes y las familias que están padeciendo esas graves y dolorosas situaciones, algunas de las cuales pueden desembocar en una psicosis luego. Los equipos nórdicos fueron pioneros en este campo, así como los australianos y algunos británicos. En España, el Programa de Prevención de las Psicosis de Cantabria (P3, www. p3-info.es) tiene seis años de existencia (se inició en el año 2000). En el caso de Catalunya, presentamos al Programa de Salud Mental del Departamento de Salud un anteproyecto, en 1999, de lo que llamábamos PAIPP (Programa de Atención Integral al Paciente con Psicosis), en el que se hacía hincapié en las actividades preventivas y de atención precoz integral (85); y en el año 2004, presentamos nuestro Proyecto de Equipo de Atención Precoz a los Pacientes con Psicosis (EAPPP), que realizó sus primeros pasos a partir de octubre de 2005 y, con el programa integrado en marcha y el personal adscrito, comenzó a rodar el 1-VI-2006.

Uno de los objetivos tanto de nuestra propuesta de PAIPP como del EAPPP era dedicar un equipo, unas actividades y un tiempo a transformar la actividad de los profesionales, los dispositivos y el pensamiento de administradores y políticos en el sentido de hacer hincapié en la prevención primaria y secundaria y no sólo en las actividades rehabilitadoras y de atención y prevención terciaria, institucional. Eso requería todo un cambio de actitud y de ideología asistencial que, en principio, pensábamos que podría tardar entre 5 y 10 años en ocurrir en la situación catalana actual. Sin embargo, la experiencia ha demostrado que fuimos conservadores en esa evolución cronológica. Hoy la necesidad de creación de equipos o programas como los que aquí vamos a exponer se ha convertido en uno de los objetivos prioritarios del Plan Director de Salud Mental y Adicciones de Catalunya. En la rapidez de ese cambio (menos de dos años), han jugado un papel preponderante, por un lado, la dinámica puesta en marcha con la creación de grupo de trabajo para la redacción del Plan Director de Salud Mental y Adicciones de la Generalitat (2006-2010), con su gran hincapié en la prevención y en las edades infantiles, al menos sobre el papel y en las intenciones. En segundo lugar, el hecho de que, por primera vez, el Institut Catalá de la Salut, decidiera en menos de un año apoyar un equipo de dichas características y dotarlo del necesario presupues- 
to. De forma tal que, en cuanto el ICS ha mostrado que puede y quiere crear y sostener un equipo de esas características, todo el resto de empresas de salud mental catalanas concertadas con el Servei Catalá de la Salut -y el propio S.C.S.-, se han lanzado a estudiar la posibilidad de poner en marcha programas y equipos piloto en este campo, tras las resoluciones de un «Grupo Operativo sobre Psicosis Incipiente» del Servei Catalá de la Salut y el Departament de Salut de la Generalitat (Grupo Operativo de Psicosis Incipiente, 2006) (40).

Como se discutió en dicho grupo de trabajo, hay dos formas de poner en marcha dichos programas. Dos formas a las que designaremos con los términos de «creación de equipos integrales piloto» $\mathrm{y}$ «programas de integración basados en las sinergias y colaboraciones entre diferentes empresas y dispositivos». Todo ello no podría entenderse si no se tiene en cuenta que en Catalunya gran parte de los dispositivos de SMiA son concertados, no propios del Servei Catalá de la Salut ni del Institut Catalá de la Salut, y que en determinadas zonas de 100.000 habitantes, como la que atiende nuestro EAPPP, puede haber hasta siete empresas diferentes haciéndose cargo de los temas de salud mental de la población. De ahí que lo que en una sanidad pública se resuelve con la creación de un equipo piloto o «demostrativo» integrado, que se pone en marcha y crea las necesarias sinergias con los equipos de otros niveles actuantes en el mismo territorio y población, en la sanidad catalana y su sistema empresarial mixto ha de tener en cuenta que esa es tan sólo una vía o posibilidad. La otra consiste en la creación de programas que han de irse integrando progresivamente basándose en acuerdos económicos y gerenciales interempresariales para compartir personal, espacios, locales, actividades, etc. En todo caso, esos «programas» o «equipos integrados» deberían cubrir las tareas y dotarse de un tipo concreto de organización sobre los cuales queríamos aportar aquí unas ideas iniciales. Se trata de las ideas y nociones que han servido de base a la creación del primer equipo catalán dedicado específicamente a estos temas, si bien para las mismas hemos tenido en cuenta tanto nuestra propia experiencia personal como las revisiones y consensos nacionales e internacionales sobre el tema $(1 ; 6-8 ; 11 ; 15 ; 28 ; 30 ; 37-49)$.

Objetivos y población-diana de los equipos de atención precoz a los pacientes con psicosis y/o de psicosis incipiente.

Los objetivos de la puesta en marcha de este tipo de equipos de atención precoz e integral a los pacientes con psicosis suelen agruparse en «generales» y «específicos». Los objetivos generales serían: a) La disminución del sufrimiento individual, familiar y social que significa tanto la duración de los pródromos del primer episodio de la esquizofrenia ( 2 a 5 años en las sociedades europeas), como la cronificación subsiguiente, que hoy parece vinculada parcialmente con el retraso en la 
ORIGINALES Y REVISIONES

ayuda integral a estos pacientes y sus familias. b) Disminuir los costos sociales de los trastornos esquizofrénicos, un resumen de los cuales aparece en las tablas 1 y 2 .

Los objetivos específicos suelen agruparse en los siguientes apartados: A) Detección precoz de los pacientes y familias sufriendo durante años los primeros síntomas, pródromos o el trema psicótico inicial. B) Atención psicosocial y psiquiátrica integral precoz. Con el fin de disminuir los índices de cronicidad, cronificación medicalizada y costos sociales del trastorno, según los datos aportados por la bibliografía pertinente. Con el fin de ampliar la escasa libertad de tratamiento del cual hoy disponen esos pacientes y sus familias. En gran parte de los casos, la realidad asistencial hace que, durante años, los únicos tratamientos que se apliquen sean tratamientos biológicos. Integrar los diferentes dispositivos terapéuticos para pacientes con psicosis de su área de influencia.

Población diana: Un esquema de ella, según nuestras perspectivas actuales, aparece en la tabla 4. Ahí especificamos que los grupos de consultantes atendidos por un EAPPP serán en realidad de esos cinco tipos. Los tres primeros, específicos para un EAPPP. Los otros dos, inevitables dada la situación de saturación de nuestros dispositivos de salud mental y el índice de desgaste profesional de bastantes de los técnicos de los mismos. El grupo MV (menores vulnerables), que nosotros consideramos de gran importancia preventiva, sólo puede ser accesible con equipos integrados que, dentro de sus capacidades técnicas, incluyan la prevención (y no sólo la asistencia) y la atención (prevención y asistencia) tanto de adultos como de niños (tablas 5, 6 y 7). Es un tema que conlleva polémica en los grupos de expertos, pero que nosotros consideramos insoslayable, máxime teniendo en cuenta las últimas orientaciones de la OMS en este campo y, en general, en el ámbito de las prioridades en la atención a la salud mental (73). En el mismo sentido, consideramos de gran valor preventivo (y social, dado el índice de maltrato doméstico en nuestras sociedades) la atención precoz de los pacientes con trastornos delirantes (celotípicos, paranoides, etc.) antes de lo que en la actualidad suele hacerse, a menudo, con la aparición pública del maltrato, la violencia, la autoexclusión social, etc.

\section{Atenciones específicas del EAPPP}

Un equipo de estas características ha de estar técnicamente preparado para realizar una serie de tareas definidas (figura 1 y tabla 8). Bastantes de ellas, aunque no todas, cuentan con una comprobación empírica suficientemente aceptable. Sin embargo, el grado o nivel de comprobación empírica de sus cualidades como técnicas es heterogéneo y no podemos aún considerar que los diversos indicado- 
res de su utilidad: eficacia, eficiencia, accesibilidad, seguridad, etc. $(61 ; 66 ; 86$; 93) se hallen totalmente comprobadas según las pruebas empíricas existentes $(17 ; 76)$. Esquematizando, en nuestro caso intentaríamos que el equipo de atención precoz a los pacientes con psicosis pudiera realizar las siguientes tareas asistenciales específicas como equipo:

1) Detección comunitaria precoz de los tres grupos de sujetos: Sujetos en riesgo de psicosis (SRP), menores vulnerables (MV) y Primeros episodios (PE) o, en otra terminología, «psicosis incipiente»: (PI). Es una actividad que debe realizarse en estrecha colaboración con los Equipos de APS, los equipos de urgencias y los equipos pedagógicos, sociales y de las instituciones comunitarias, etc. Incluso este tipo de actividades debería ser evaluado para determinar la posible eficacia y eficiencia de este tipo de equipos. Indicadores para la evaluación podrían ser del tipo línea de base de incidencia en servicio durante el último año o los años siguientes, tasas de detección confirmadas con SCAN, PANSS, ERIE-IRAOS y FETZ u otros instrumentos psicopatológicos, variabilidad en la procedencia de los sujetos derivados, etc.

2) Visitas a domicilio, en el Centro de Atención Primaria o en el propio Equipo para establecer el vínculo terapéutico (con el paciente o probable paciente y con la familia). Los sistemas de evaluación de esta actividad podrían basarse en la comprobación diagnóstica mediante escalas y entrevistas estándar (ERIE, SCAN, PANSS), índices de falsos positivos/falsos negativos con el fin de poder reevaluar los conceptos y metodología, índice de visitas a domicilio y/o CAP que dan lugar a un «vinculo terapéutico estable», etc.

3) Intervenciones psicoterapéuticas y psicosociales. En el caso de los «falsos positivos», que no resulten pacientes psicóticos o SRP, habría que aplicar medidas psicosociales de urgencia, si se precisaran, y vincularlos con su Centro de Atención Primaria y/o de salud mental correspondiente. En los casos que resulten diagnosticados de psicosis, primer episodio, o de SRP, habría que aplicar el tipo de tratamiento que hemos resumido con el acrónimo TAN-PFC (tratamiento adaptado a las necesidades del paciente y su familia en la comunidad), compuesto por los siguientes elementos: a) Tratamiento psicoterapéutico «adaptado a las necesidades» (del paciente y la familia). Seleccionado, en función del paciente y su familia y en función de la preparación del equipo, varias de las siguientes técnicas $(7 ; 11 ; 16 ; 39 ; 83 ; 87-91)$ talleres psico-educativos multifamiliares y/o grupo psico-educativo, psicoterapia individual y/o psicoterapia grupal de los pacientes, terapia de resolución de problemas y grupo de habilidades sociales, atención psicoterapéutica familiar (psicoanalítica o sistémica) y/o grupo paralelo de familiares de pacientes y/o grupo multifamiliar, ayuda psicoterapéutica a la pareja parental en el caso de MV, centrada en los propios padres como pacientes o bien aceptando (provisionalmente) el modelo de «atención a los 
ORIGINALES Y REVISIONES

padres a través del hijo» (92); b) Ayudas psicosociales. En colaboración con el trabajo, la institución escolar, los servicios sociales e instituciones sociales, etc. En el propio equipo habría que poner en marcha ayudas del tipo «terapia de resolución de problemas sociales» y «grupo de habilidades sociales». c) Tratamiento psicofarmacológico con dosificaciones prudentes que permitan realizar el resto de la terapia combinada, según el modelo expuesto por ejemplo por Alanen, Cullberg o Tizón $(2 ; 11 ; 87 ; 93)$.

Los sistemas de evaluación de estos tres tipos de actividades son mucho más conocidos y vienen resumidos por ejemplo en los trabajos de Edwards y McGorry, Martindale y otros (7), Read y otros (48), Olsen y Rosenbaum $(8 ; 17 ; 76)$, etc. Sin embargo, para asegurar la eficacia y eficiencia de los EAPPP habría que adaptar esos sistemas a nuestras realidades, tanto sociales como asistenciales. Por ejemplo, para evaluar la eficacia de las medidas psicosociales en casos no-psicóticos (SRP) podría utilizarse las tasas de vinculación con el CAP o USM, GHQ y escalas psicopatológicas, escalas de calidad de vida tipo Lancanshire (94), etc. En los casos PE o PI, la eficacia y demás indicadores de calidad podrían evaluarse a través de indicadores funcionales, tales como los índices de vinculación al CAP o a la USM, índice de aceptación de los diversos tratamientos individuales, índice de aceptación de los diversos tratamientos grupales, evolución psicopatológica (Escalas de calidad de Vida, PANSS, Índices de adaptación social, etc.), tasas de ingresos hospitalarios grupo de intervención/grupo control, dosis psicofarmacológicas y costes en este y otros capítulos, etc.

4) Delimitación, cuanto más temprana mejor, de los grupos globales de pacientes con trastornos psicóticos o pródromos. Por las experiencias anteriores, a este tipo de equipo probablemente acudirán cinco tipos de pacientes $(6 ; 8-9$; $11 ; 17 ; 76 ; 79 ; 97-102)$. En primer lugar, tres grupos de pacientes con «psicosis delirante» (PPD): a) Pacientes «psicóticos» ya cronificados, que acuden al EAPPP por error o por desvinculación con respecto a otros dispositivos de la red asistencial correspondiente. Con ellos habría que intentar realizar la vinculación con la USM y el CAP correspondientes. b) Pacientes con psicosis cuya evolución hacia la cronificación está avanzada. Por criterios de eficiencia y de coherencia con el resto del circuito asistencial, el EAPPP debería intentar la vinculación de la familia y el paciente con el Centro de Salud Mental correspondiente y, si el CSMA lo solicita, el tratamiento familiar durante un máximo de un año. Debería también asegurar la coordinación inter-equipos para intentar aplicar el programa tipo PAIPP a cargo de los equipos de su territorio (85). c) En el caso de pacientes y familias en pródromos, trema o inicio del primer episodio psicótico (estimación de los PE: 30-40 \% de los detectados) habría que intentar aplicar el programa terapéutico «adaptado a las necesidades» del paciente y su familia en la comunidad. 
Además, acudirán otros dos grupos: d) El equipo debería atender preferentemente a quienes llamamos «Sujetos en riesgo de psicosis» (SRP). El grupo de individuos sometidos a importantes factores de riesgo y que muestran síntomas más o menos inespecíficos de psicosis (crisis de ansiedad, fobias sociales, consumo de sustancias sin patrón o con patrones muy irregulares, síntomas esquizotípicos y esquizoides, etc.). En el caso de este grupo, las tareas de un EAPPP deberían ser las de un «Seguimiento terapéutico», con aplicación de protocolos «adaptados a las necesidades». Tras el seguimiento adecuado, con una limitación temporal en nuestro caso de entre 2 y 5 años como máximo, se debería proceder a realizar el alta o derivación hacia los equipos de SM y APS de la red según evolución en caso de no avanzar hacia la psicosis. La dificultad del enfoque de la atención de este grupo es que aún sabemos poco de su delimitación exacta, los sistemas y medios para su detección o los índices de evolución hacia psicosis. e) Un quinto grupo de sujetos debería ser buscado específicamente por los EAPPP de vocación más preventiva. Se trata de los «menores vulnerables» (MV) por acumulación de factores de riesgo, o por síntomas y trastornos específicos: hijos de psicóticos, niños que acumulan FR y SA en las revisiones del programa del «niño sano» (103), niños detectados por cuestionarios o entrevistas ad hoc tipo CBCL o LISMEN, etc. (19; $20 ; 60 ; 104)$ (tabla 4). En este grupo, el EAPPP debería asegurar que, con la participación central del Equipo de salud mental infanto-juvenil y/o de trastornos mentales graves en la infancia, se pusieran en marcha con estos sujetos los programas preventivos necesarios y previamente definidos. El sistema de evaluación de estas actividades «según grupos de consultantes» debería estar fundamentado en el uso de escalas de evaluación y entrevistas estructuradas o semiestructuradas tipo PANSS, SCAN, ERIE-IRAOS, y los criterios CAARMS, SIPS o EASE, calidad de vida, etc. y, al tiempo, de las tasas de vinculación al EAPPP de cada de los grupos, según sus características psicopatológicas, así como de las tasas posteriores de vinculación y uso del resto de los servicios de la red de salud mental (primarios, secundarios o terciarios).

5) Seguimiento clínico y mediante protocolos de investigación empírica de la cohorte «Grupo de intervención» (entre tres y cinco años). El EAPPP realizará el tratamiento con cada paciente y familia durante 1 a 3 años (media, 2 años). La incidencia en servicio estimada, en nuestros cálculos actuales, es de 10 a 30 nuevos pacientes y familias/año/100.000 habitantes en el caso del grupo PE y entre 30 y 150 del grupo SRP. Para este apartado, el sistema de evaluación debería basarse en la comparación grupo control versus el grupo de intervención, por ejemplo, en los siguientes apartados: Tasa de ingresos en dispositivos hospitalarios, secundarios y terciarios, medicación, dosis, costos, costes de otros tratamientos, escalas de calidad de vida, número de episodios psicóticos agudos o subagudos (ERIE-IRAOS), etc. 
ORIGINALES Y REVISIONES

6) Actividades de Coordinación: Sería imprescindible una coordinación estrecha y un trabajo en red con el Centro de Atención Primaria y el Centro de Salud Mental correspondientes, el Circuito asistencial de adultos correspondiente, equipo de TMS o PSI correspondiente, Equipo de PAIPP o TMS si existiera, el Grupo de trabajo correspondiente del Plan Director de Salud Mental y el Consell Asesor de Salud Mental, los dispositivos de enseñanza y el Programa de Salut i Escola, los dispositivos de SS, etc. (Tabla 9). Pero el EAPPP debería realizar actividades en los medios de comunicación, espacios públicos y empresas de publicidad, según el modelo australiano, finlandés, holandés o alemán. Los indicadores para la evaluación en este apartado podrían provenir de índices como los siguientes: tasa de reuniones/paciente atendido, índice de reuniones con planes asistenciales concretos para pacientes concretos/número de reuniones, actas documentadas de cada reunión, etc.

7) Puesta en marcha con estas familias del «Programa preventivo para hijos de pacientes con diagnóstico de TMS» (18-20; 92).

8) Actividades de formación y supervisión: La formación específica y la supervisión son elementos básicos no sólo para la calidad de la asistencia, sino para asegurar disminuir en lo posible el inevitable «desgaste profesional» del personal de este tipo de equipos. En ese sentido, habría que asegurar que los EAPPP, por separado o agrupados, pudieran realizar los siguientes tipos de supervisiones y actividades formativas: supervisiones de la orientación clínica global de los pacientes, según el sistema de «clínico referente» (92), supervisiones de las terapias grupales, supervisiones de las psicoterapias individuales, supervisiones de las terapias familiares, supervisiones de las técnicas psico-educativas, cognitivas y de resolución de problemas, formación específica y estancias en servicios similares escandinavos, británicos, alemanes, norteamericanos o australianos, etc.

9) Actividades docentes: La docencia en este tipo de equipos y dispositivos tiene un doble papel: por un lado, asegurar la difusión de la formación y experiencia en este tipo de tareas y asistencia; por otro, ayudar a disminuir el «desgaste profesional» de los miembros de los equipos. Además, tal vez un objetivo a medio plazo sería avanzar hacia una formación integrada y específica, por ejemplo, según el modelo finlandés (2): un sistema de formación integrado de postgrado, interdisciplinario, sobre «Atención Integral al Paciente Psicótico». Tal programa docente e integrado en la «carrera profesional» podría pivotar en el personal docente de diversos EAPPP, en profesorado universitario y/o del Instituto de Estudios de la Salud y en diversos institutos de formación continuada interesados por el tema.

10) Actividades de Investigación: Los primeros equipos de estas características deben concebirse asentados directamente sobre la investigación continua- 
da de su trabajo y desarrollo. Para ello, habrá que introducir el personal adecuado, a ser posible formado previamente en el ámbito de investigación comunitaria de los trastornos psicóticos. La inclusión de la investigación como tarea indispensable se fundamenta en la necesidad de evaluar su eficacia, eficiencia y demás indicadores de calidad, pero también como otro de los medios para reducir el «desgaste profesional» del personal de esos equipos. Se trataría de que éstos realizaran al menos tres tipos de investigaciones: a) «longitudinales» del funcionamiento del equipo y el proyecto; b) específicas, tales como la de evolución de la cohorte en tratamiento a lo largo de los años en comparación con el grupo control y la evolución de los hijos de dichos pacientes en comparación con éste; c) sobre la realidad de la vida cotidiana de los pacientes de estas características en nuestras sociedades.

\section{Necesidades de personal}

Las investigaciones y revisiones europeas $(2-7 ; 28,95-96)$, que hemos podido corroborar y contrabalancear con nuestras propias investigaciones (19), muestran que la incidencia en servicios de alta accesibilidad de los trastornos psicóticos es de 1 a 3 nuevos casos por 10.000 habitantes, con un máximo de 6 por 10.000 habitantes. Eso significaría que, en una población de 100.000 habitantes, geodemográficamente delimitada, el EAPPP habría de hacerse cargo inicialmente de unos 10 a 30 nuevos casos de PE /año (ya que cerca de la mitad de los otros pacientes detectados probablemente se hallarían en un estadio de evolución avanzado con respecto a su trastorno).

Teniendo en cuenta los datos sobre dedicación horaria por paciente, que hay que incrementar en al menos el 50\% con la dedicación a coordinación y trabajo en red, formación, supervisión e investigación, así como el tipo e intensidad de la cartera de servicios ofertados por el EAPPP (Tablas 8 y 10), estimamos necesaria la siguiente dotación de personal para iniciar este Programa piloto: un director, 1-2 psicólogos con formación psicoterapéutica específica (psicoanalítica y cognitivo-conductual), 1-2 psiquiatras con formación psicoterapéutica y experiencia en el tratamiento de pacientes psicóticos, 1 trabajador social especializado, 2 enfermeros especializados en visitas domiciliarias, 1 secretaria en cada turno, y un técnico de salud o de investigación psicosocial a media jornada (personal calculado para una zona geodemográficamente delimitada de 100.000 habitantes).

Ya en Catalunya se ha discutido sobre el dilema «equipos específicos» o «programas específicos integrados en Centros de Salud Mental». Existen argu- 
ORIGINALES Y REVISIONES

mentos para apoyar ambas alternativas, máxime en un panorama gerencial como el catalán, en el que se dan más de sesenta empresas diferentes atendiendo a la salud mental, de tal forma que constituir un equipo coherente, integrado y diferenciado puede chocar con los intereses gerenciales diferenciados de las mismas: ello parece que aporta argumentos a la idea de que es mejor crear programas integrados en los CSM del sector o integrando personal procedente de diferentes equipos y niveles asistenciales (CSM, servicios de ingreso, hospital de día, etc.) para facilitar la aceptación del funcionamiento del tipo de atención aquí propuesto dentro de la red. Pero es dudoso que puedan constituirse equipos bien integrados, proactivos y estables por la vía de la integración progresiva a partir de experiencias y empresas diferentes. Máxime si tenemos en cuenta que serán equipos que han de atender a trastornos cuyo problema fundamental está asimismo en la integración (del self y con la comunidad): la tendencia a la escisión y disociación que promueve el contacto continuado con la psicosis facilita el desarrollo de esas mismas tendencias en los equipos asistenciales -lo cual se tiene poco en cuenta en el replanteamiento de la atención a los pacientes con psicosis-. Por eso, si las experiencias e historias institucionales son diferentes, puede resultar algo más difícil proteger adecuadamente a medio plazo las actividades específicas, dedicación y protección del desgaste profesional del personal integrado en este tipo de «programas». Con todo, esa discusión (menos relevante en lugares donde existe una empresa única para proporcionar servicios de salud mental), ha sido revisada a raíz de la muerte a manos de un paciente del psiquiatra estadounidense Wayne Fenton, antiguo investigador del NIHM. La revisión realizada por Smith y Shiers (77) es aclaratoria, pues se necesitan equipos integrados, dedicados a este tipo de programas y con personal formado, protegido y motivado, algo difícil de gestionar en un CSMA habitual o sólo integrando personal de procedencia diversa $(6 ; 30 ; 77)$.

En cualquier caso, dadas las diferencias en incidencia, prevalencia, frecuentación, integración social, evolución y demás características de los pacientes diagnosticados de «psicosis» o incluso de «esquizofrenia» en diversos medios geodemográficos $(20 ; 48)$, el sistema de funcionamiento debería ser como «Equipo o Programa Piloto». El desarrollo del equipo, su extensión a otras zonas geodemográficas, y su composición y desarrollo interno deberían estar sujetos a las especificaciones proporcionadas por las evaluaciones y autoevaluaciones del mismo.

Asimismo, la adscripción del personal al Equipo, dado su alto índice de «desgaste profesional» potencial, debería ser temporal. El personal debidamente formado y que cumpliera el perfil, podría solicitar su adscripción al programa durante un período de dos años renovables por ambas partes (Dirección y 
Gerencia del programa, por un lado, y profesional, por otro). Porque, tal vez a diferencia de propuestas que se han ido realizando desde los años sesenta del pasado siglo acerca de la «reforma de la atención a las psicosis», hoy sabemos algo más acerca de la gravedad de los trastornos de los cuales estamos hablando $\mathrm{y}$, también, sobre las limitaciones y repercusiones que su cuidado obra sobre los equipos y profesionales que nos dedicamos a ello. Sabemos algo más acerca de cómo un tipo de relación que se halla en la base de muchos trastornos psicóticos, el tipo de relación al que he llamado «simbiótico-adhesivo», es difícilmente modificable si no es con un trabajo integral, intensivo y precoz. Entre otras cosas, porque conlleva el que esos pacientes o potenciales pacientes tengan una gran tendencia a «perderse en los intersticios sociales y en sus propios repliegues psíquicos», tendencia que a veces no ha sido estudiada y atendida con la gravedad que comporta -y menos aún, en cómo repercute en actitudes y organizaciones de los equipos cuidantes-. En suma, estamos avanzando en un tipo de trabajo que en absoluto es ni va a ser la panacea para solucionar estos graves sufrimientos de pacientes, familias y equipos. Se trata tan sólo de un modelo de trabajo que merece que le dediquemos actualmente al menos una parte de nuestra experiencia y capacidades profesionales y personales.

Tabla 1

El coste de la esquizofrenia

\begin{tabular}{ccc}
\hline País & Estudio & Coste \\
\hline & & 2 '5\% de los gastos directos de Salud $=19.000$ \\
\multirow{3}{*}{ USA } & APA & (millones de dólares en 1990). \\
& (M. L. Bourgeois [105]) & $10 \%$ de las invalideces totales. \\
& & $25 \%$ de los esquizofrénicos sin alojamiento. \\
& $25-50 \%$ de los pacientes, sin tratamiento alguno. \\
\hline
\end{tabular}

$12,5 \%$ de los gastos de salud (cerca del $10 \%$ PIB).

82.600 Francos/año (2-3 M de ptas.)

Francia F. Rouillon, y otros, 1997 (105)

Menor que en Suecia y similar a USA

Una recaída de un esquizofrénico cuesta lo mismo que un tratamiento de mantenimiento de por vida.

\begin{tabular}{cll}
\hline UK & (Según M. L. Bourgeois [105]) & $\begin{array}{l}\text { Gastos menores (hay } 3 \text { veces menos psiquiatras que } \\
\text { en Francia) }\end{array}$ \\
\hline Suecia & B. Wistedt,1992 (105) & Una recaída cuesta 2.000 kr. (1992). \\
\hline & & Coste familiar objetivo anual (Ptas. 1997): \\
& & 115.318 ptas./mes en Gavá \\
España & PSICOST $(106 ; 107)$ & 11.102 en Burlada \\
& & Sin contar los gastos $\ll$ «top-down»: \\
& & Por ej. Costes NNLT/paciente/año $=2.000 €$ de media \\
\hline
\end{tabular}




\section{ORIGINALES Y REVISIONES}

Tabla 2

El sufrimiento de la esquizofrenia

\begin{tabular}{ll}
\hline Suicidio & 3-13\% en los dos primeros años \\
\hline Prevalencia puntual & $\begin{array}{l}\text { Finlandia: 0'1-0'8 \% (Y. Alanen [2]) } \\
\text { Sant Martí (BCN): 0'37-0'7 }\end{array}$ \\
\hline \multirow{2}{*}{ Prevalencia vida } & $\begin{array}{l}\text { 0'7-1 \% } \\
\text { (mayor en los estudios ECA norteamericanos) }\end{array}$ \\
\hline \multirow{2}{*}{$\begin{array}{l}\text { Incidencia en Servicio } \\
\text { (por 10.000 habitantes) }\end{array}$} & UK: 0'7-1'4 \\
& Finlandia: 1'1-1'4 \\
& $\begin{array}{l}\text { Finlandia (junto con los trastornos esquizofreniformes): 1'6-2'0 } \\
\text { Sant Martí (Barcelona): 3'56-5'85 }\end{array}$ \\
\hline Incidencia de Tr. Mentales en servicio & $\begin{array}{l}\text { Finlandia: 0'4 \% } \\
\text { Sant Martí (Barcelona): 1'2\% }\end{array}$ \\
\hline Pensiones para pacientes (Finlandia y UK) & 10\% del total \\
\hline
\end{tabular}

Tabla 3

Incidencia en servicio de esquizofrenia y otras psicosis (2001-2003), según el «Registro de Casos» de la USM Sant Martí-La Mina, fichero «Siglo XXI».

Nuevos casos. Población: 105.516 habitantes desde el 2001

\begin{tabular}{|c|c|c|c|c|c|c|}
\hline & & $\begin{array}{l}\text { Adultos } \\
\text { La Verneda }\end{array}$ & $\begin{array}{l}\text { Menores } \\
\text { La Verneda }\end{array}$ & $\begin{array}{l}\text { Adultos } \\
\text { La Mina }\end{array}$ & $\begin{array}{l}\text { Menores } \\
\text { La Mina }\end{array}$ & Total \\
\hline \multirow{2}{*}{2001} & Esquizofrénicos & 6 & 0 & 3 & 0 & 9 \\
\hline & Otras psicosis & 8 & 0 & 7 & 2 & 17 \\
\hline \multirow{2}{*}{2002} & Esquizofrénicos & 8 & 0 & 4 & 0 & 12 \\
\hline & Otras psicosis & 8 & 2 & 4 & 1 & 15 \\
\hline \multirow{2}{*}{2003} & Esquizofrénicos & 10 & 0 & 1 & 0 & 11 \\
\hline & Otras psicosis & 14 & 0 & 1 & 1 & 16 \\
\hline \multirow{2}{*}{$\begin{array}{c}\text { TOTALES } \\
\text { «SASPE } \\
\text { Siglo XXI» } \\
(2001-2003)\end{array}$} & Esquizofrénicos & 24 & 0 & 8 & 0 & $\begin{array}{l}\quad 32 \\
\text { (3'03 por diez mil= } \\
\text { 1'01 anual) }\end{array}$ \\
\hline & Otras psicosis & 30 & 2 & 12 & 4 & $\begin{array}{l}\quad 48 \\
\text { (4'54 por diez mil = } \\
\text { 1'51 anual) }\end{array}$ \\
\hline
\end{tabular}


Tabla 4

Grupos de población-Diana y actividades correspondientes

\begin{tabular}{|c|c|}
\hline $\begin{array}{l}\text { - Psicosis Incipiente ( } 1 \text { er episodio hace un año) }^{\text {er }} 10-30 \text { casos/año/100.000 habs. }\end{array}$ & - Programa terapéutico completo (PTI y TANC) \\
\hline $\begin{array}{l}\text { - SRP o EMAR: } \\
\text { - Estimación: } 200-150 \% \text { de los } \\
\text { derivados en primer episodio } \\
\text { - } 50-60 \text { casos/año/100.000 habs. }\end{array}$ & $\begin{array}{l}\text { - Programa terapéutico adaptado a sus necesidades } \\
\text { del paciente y su familia en la comunidad (TANC) }\end{array}$ \\
\hline $\begin{array}{l}\text { - MV (Menores Vulnerables): } \\
\text { Adolescentes o Niños con acumulación } \\
\text { de Factores de Riesgo. } \\
\text { (Grupo difícilmente cuantificable: } \\
\text { depende de la eficacia preventiva) }\end{array}$ & $\begin{array}{l}\text { - Programas preventivos, seguimiento, coordinación } \\
\text { con los equipos correspondientes... }\end{array}$ \\
\hline $\begin{array}{l}\text { - Tr. Psicóticos o «Esquizofrénicos» con } \\
\text { evolución hacia la cronificación, no PE } \\
\text { (probablemente, hasta el 30-40\% } \\
\text { de los derivados) }\end{array}$ & $\begin{array}{l}\text { - Vinculación de la familia y el paciente } \\
\text { con la CSMA correspondiente y tratamiento } \\
\text { familiar durante un máximo de un año. } \\
\text { - Coordinación para intentar aplicar el programa } \\
\text { PTI a cargo de los equipos de su territorio. }\end{array}$ \\
\hline $\begin{array}{l}\text { - TR. Psicóticos o Esquizofrénicos } \\
\text { ya cronificados } \\
\text { (Difícil de cuantificar: } \\
\text { depende de la eficacia asistencial) }\end{array}$ & $\begin{array}{l}\text { - Vinculación con el CSMA y el CAP } \\
\text { correspondientes. }\end{array}$ \\
\hline
\end{tabular}

Tabla 5

Criterios de derivación desde los CSMIJ o desde pediatría

\begin{tabular}{ll}
\hline \multicolumn{1}{c}{$\begin{array}{c}\text { CUADROS CLÍNICOS SASPE } \\
\text { en la PUBERTAD y ADOLESCENCIA }\end{array}$} & \multicolumn{1}{c}{$\begin{array}{c}\text { Programas asistenciales } \\
\text { o preventivos específicos }\end{array}$} \\
\hline - ESQUIZOFRENIA & \\
- Trastorno esquizofreniforme & - Hijos de pacientes con Psicosis Delirante. \\
- Trastorno esquizoafectivo & - Adolescentes postautistas, psicóticos, etc. \\
- Trastorno psicótico breve & - Complementos para sus terapéuticas habituales. \\
- Trastorno psicótico compartido & - Dudas diagnósticas sobre padres, hijos, familia. \\
- Trastorno psicótico debido a & Niños o chicos con acumulación de FR \\
enfermedad médica & y SA en el Programa del Niño Sano o en el LISMEN. \\
- Trastorno psicótico inducido & Niños o chicos con SA en el Protocolo \\
por sustancias & de SM del Programa del Niño Sano. \\
- Trastorno psicótico no especificado & \\
\hline
\end{tabular}


ORIGINALES Y REVISIONES

Tabla 6

Criterios para la adscripción al gupo SRP

(Cada equipo utilizará los criterios A-B-C o bien el D en función de su formación y capacidades de investigación empírica).

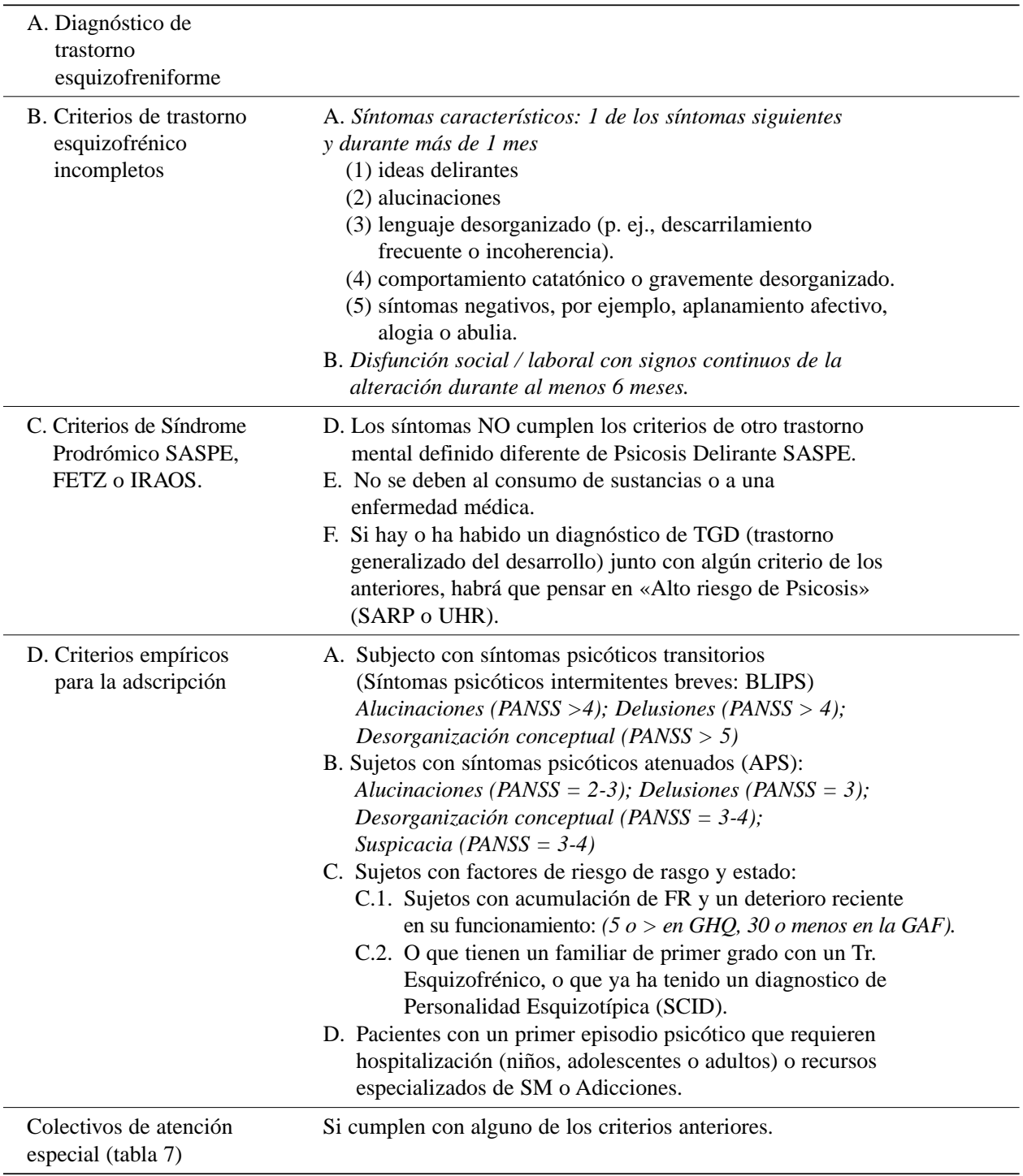


Tabla 7

Colectivos de atención especial

Personas marginales desvinculadas de las redes profesionales.

Abuso de sustancias, desorganizado, atípico, con múltiples ingresos en distintas instituciones.

Inmigrados sin red social.

Personas víctimas de abusos sexuales en la infancia.

Personas con antecedentes previos de trastornos psiquiátricos inespecíficos.

Personas con tentativas de suicidio.

Personas que provienen de familias desestructuradas multiproblemáticas.

Otros grupos de Prevención del Plan Director de Catalunya 2006-2010.

Gran prematuridad y complicaciones obstétricas.

Padres con trastornos mentales graves.

Madres en situación de riesgo psicosocial.

Maltrato y abusos en la infancia.

Fracaso escolar reciente o abandono de la escolaridad.

Adicciones en la infancia y la pubertad.

TGD.

Trastorno Disocial.

Retraso mental.

TDAH.

Depresión o TOC en la infancia.

Tabla 8

Actividades del EAPPP

- Detección comunitaria precoz.

- Visitas a domicilio / en el CAP para establecer el vínculo terapéutico.

- Intervenciones psicoterapéuticas y psicosociales.

- Talleres psicoeducativos multifamiliares.

- Psicoterapia individual.

- Grupo psicoeducativo.

- Psicoterapia Grupal de los pacientes.

- Grupo paralelo de Familiares de Pacientes.

- Terapia de resolución de problemas y grupo de habilidades sociales.

- Atención psicoterapéutica familiar (psicoanalítica o sistémica).

- Grupo multifamiliar.

- Ayuda psicoterapéutica a la pareja parental (o atención a los padres a través del hijo).

- En colaboración con el trabajo, institución escolar, servicios sociales, o instituciones.

- Terapia de resolución de problemas sociales y Grupo de Habilidades sociales.

- Intervenciones psicofarmacológicas adaptadas a las «terapias combinadas» (93).

Con dosificaciones prudentes que permitan realizar el resto de la terapia combinada.

- Atenciones específicas relacionadas con la delimitación temprana de los grupos de psicóticos.

- Seguimiento de la cohorte «Grupo de intervención».

- Actividades de Coordinación.

- «Programa preventivo para hijos de pacientes con diagnóstico de TMS»y otros.

- Actividades de formación y supervisión.

- Actividades docentes.

- Actividades de Investigación. 


\section{ORIGINALES Y REVISIONES}

Tabla 9

Actividades de Coordinación

1. Con la red de SM:

- Con el CAP y el CSMA-CSMIJ correspondientes.

- El Circuito asistencial de adultos correspondiente.

- Equipo de TMS o PSI correspondiente.

- Equipo de PAIPP si existiera.

- Grupo de trabajo correspondiente del Plan Director de Salud Mental y el Consell Asesor de Salud Mental.

2. Con los Equipos de APS: Medicina de Familia y Pediatría.

3. Otras redes y departamentos:

- Los dispositivos de enseñanza y EAP de la zona.

- El Programa de Salut i Escola.

- Los dispositivos de S. S.

- Dispositivos de Justicia.

4. Actividades con la comunidad y los medios de comunicación, espacios públicos y empresas de publicidad, según el modelo australiano, danés, finlandés o alemán.

Tabla 10

«Atención Directa» que supondría cada paciente atendido por EAPPP

\begin{tabular}{lll}
\hline Tipo de terapia & $\begin{array}{l}\text { Frecuencia de las sesiones / } \\
\text { entrevistas }\end{array}$ & $\begin{array}{l}\text { Tiempos por semana equivalentes } \\
\text { por paciente en horas o fracciones }\end{array}$ \\
\hline Entrevistas familiares & Quincenal & 0,5 \\
Psicoterapia individual & Dos sesiones por semana & 2 \\
Psicoterapia grupal & Semanal, 4-8 pacientes & 0,25 \\
Grupo multifamiliar & $\begin{array}{l}\text { Quincenal, 4 pacientes } \\
\text { y sus familias }\end{array}$ & 0,25 \\
Grupos Psicoeducativos & Semanal, 4 pacientes & 0,25 \\
$\begin{array}{l}\text { Terapias cognitivas, } \\
\text { de Rehabilitación y TRP }\end{array}$ & Semanal & 1 \\
Entrevista psiquiátrica & Quincenal & 0,5 \\
Tiempos TOTALES & & 4,75 horas/semana/paciente o familia (asist. directa) \\
\hline
\end{tabular}


Figura 1

Evolución de una psicosis y actividades correspondientes de los EAPPP

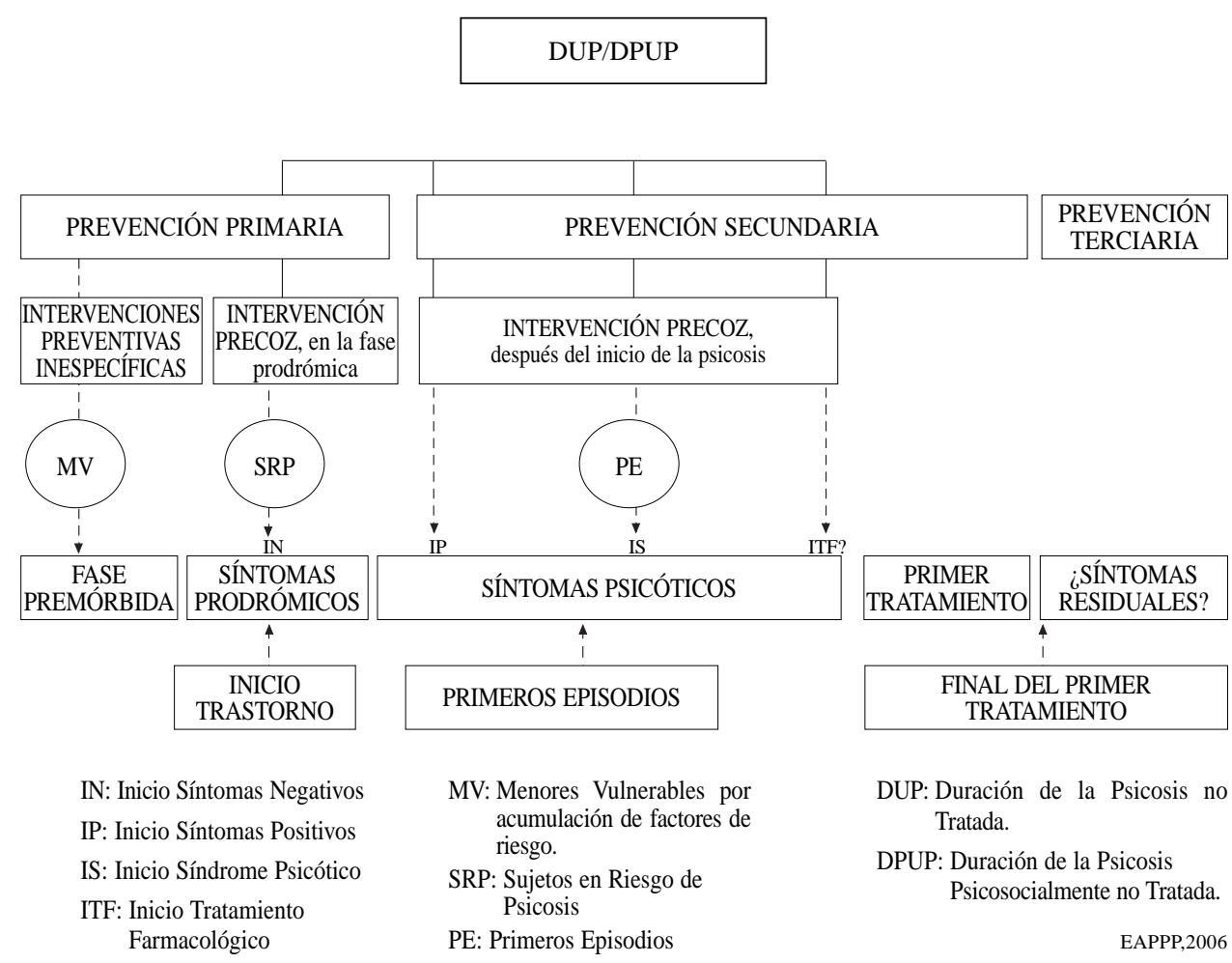


ORIGINALES Y REVISIONES

\section{BIBLIOGRAFÍA:}

(1) HäFner, H.; Der Heiden, W., «Epidemiology of Schizophrenia», Can J. Psychiatry, 1997, 42 (2), pp. 139-51.

(2) Alanen, Y. O., y otros, «The Finish Integrated Model for early Treatment of Schizophrenia and Related Psychoses», en MARTindale, B., y otros (eds.), Psychosis: Psychological Approaches and their Effectiveness, Londres, Gaskel-ISPS, 2000, pp. 235-266.

(3) Birchwood, M., y otros, «Depression, Demoralisation and Control over Psychotic Illness», Psychological Medicine, 1993, 23, pp. 387-395.

(4) BIRChWOOD, M., «The Critical Period for Early Intervention», en BIRCHWOOD, M.; Fowler, D.; JACKSON, C. (eds.), Early Intervention in Psychosis: A Guide to Concepts, Evidence and Interventions, Chichester, Wiley, 2000, pp. 28-63.

(5) Birchwood, M.; Spencer, E.; McGovern, D., «Schizophrenia: Early Warning Signs», Advances in Psychiatric Treatment, 6, 2000, pp. 93-101.

(6) BIRCHWOOD, M., «Intervención temprana en psicosis: investigación, práctica clínica y reforma de los servicios en el Reino Unido», en VÁzQuez-BARQUero, J. L.; CresPo, B.; HerRán, A., Las fases tempranas de las enfermedades mentales: Psicosis, Barcelona, Masson, 2005, pp.145-159.

(7) Martindale, B.; Bateman, A., y otros (eds.), Psychosis: Psychological Approaches and their Effectiveness, Londres, Gaskel-ISPS, 2000.

(8) EdWARd, J.; McGorry, P., La intervención precoz en la Psicosis: Guía para la creación de servicios de intervención, Madrid, Fundación para la Investigación y el Tratamiento de la Esquizofrenia y otras psicosis, 2004.

(9) VÁzquez-Barquero, J. L.; Crespo, B.; Herrán, A., Las fases iniciales de las enfermedades mentales: Psicosis, Barcelona, Masson, 2004.

(10) TızóN, J. L., «Resiliencia y contención en pacientes diagnosticados de esquizofrenia», Archivos de psiquiatría, 2006, 69 (2), pp. 81-84.

(11) Alanen, Y. O., Schizophrenia: Its Origins and Need-Adapted Treatment, Londres, Karnac, 1999.

(12) Jobe, T. H.; Harrow, M., «Long-Term Outcome of Patients With Schizophrenia», Can J. Psychiat., 2005, 50, 4, pp. 892-900.

(13) Jones, P.; CAnnon, M., «The New Epidemiology of Schizophrenia», Psychiatr. Clin. North Am., 1998, 21 (1), pp. 1-25.

(14) Marcelis, M., y otros, «Urbanization and Psychosis: a Study of 1942-1978 Birth Cohorts in The Netherlands», Psychol. Med., 1998, 28 (4), pp. 871-79.

(15) MCGORRY, P. D., y otros, «EPPIC: An Evolving System of early Detection and Optimal Management», Schizophrenia Bulletin, 1996, 22 (2), pp. 305-326.

(16) McGorry, P. D., «Psychotherapy and Recovery in early Psychosis: a Core Clinical and Research Challenge», en MARTINDALE, B., y otros (eds.), Psychosis: Psychological Approaches and their Effectiveness, Londres, Gaskel-ISPS, 2000, pp. 266-292.

(17) Olsen, K. A.; Rosenbaum, B., «Prospective Investigations of the Prodromal State of Schizophrenia», Acta Pscyhiat. Scan., 2006, 113, pp. 247-272.

(18) Tizón, J. L., Repercusiones sobre el niño de los trastornos mentales de sus progenitores y cuidadores, Formación Médica Continuada, Barcelona, 2005, 12 (4), pp. 220-236.

(19) TIzÓN, J. L., y otros, «La esquizofrenia en atención primaria: El Estudio SASPE (Señales de alerta y síntomas prodrómicos de la esquizofrenia en APS). Protocolo de una investigación empírica», Atención Primaria, 2004, 34 (9), pp. 493-498. 
(20) TIZÓN, J. L., y otros, «Hijos de pacientes con psicosis en el Proyecto SASPE: Investigar para cuidar un futuro comprometido», Archivos de Psiquiatría, 2006, 691, pp. 59-80.

(21) Torrey, E. F.; Bowler, A. E.; ClARK, K., «Urban Birth and Residence as Risk Factors for Psychoses: An Analysis of 1880 Data», Schizophr. Res., 1997, 25 (3), pp. 169-6.

(22) VÁzquez-BARquero, J. L., y otros, «Factors Implicated in the "Routes of Care" in Mental Health», Actas Luso Esp. Neurol. Psiq. Cienc. Afines, 1993, 21 (5), pp. 88-203.

(23) VÁzQuez-BARquero, J. L., y otros, «Pathways to Psychiatric Care in Cantabria», Acta Psychiatr. Scand., 1993, 88 (4), pp. 229-4.

(24) VÁzquez-BARquero, J. L., y otros, «The Cantabria First Episode Schizophrenia Study: a Summary of General Findings», Acta Psychiat. Scand., 1995, 91, pp. 156-62.

(25) VÁzquez-BARquero, J. L., y otros, «Cantabria First-Episode Schizophrenia Study: Three-Year Follow-Up», British Journal of Psychiatry, 1999, 174, pp. 141-9.

(26) Woods, S. W., y otros, «Characteristics of Participants and Nonparticipants in Medication Trials for Treatment of Schizophrenia», Psychiatric Services, 2000, 51 (1), pp. 79-84.

(27) McGlashan, T. H., «Duration of Untreated Psychosis in First-Episode Schizophrenia: Marker or Determinant of Course?», Biological Psychiatry, 1999, 46, pp. 899-907.

(28) HÄFNER, H., y otros, «Causes and Consequences of the Gender Difference in Age at Onset of Schizophrenia», Schizophr. Bull., 1998, 24 (1), pp. 99-113.

(29) JohANNESSEN, J. A., y otros, «Early Intervention in Psychosis: The TIPS Project, a MultiCentre Study in Scandinavia», en Martindale, B., y otros (eds.), Psychosis: Psychological Approaches and their Effectiveness, Londres, Gaskel-ISPS, 2000, pp. 210-235.

(30) Johannessen, J. A., «The Development of early Intervention Services», en ReAD, J.; Mosher, L.; Bentall, R. (eds.), Models of Madness: Psychological, Social and Biological Approaches to Schizophrenia, Londres, Brunner-Routledge, 2004.

(31) Al Mousawi, A. H.; Dunstan, F. D., «Changes in the Risk of Schizophrenia in Scotland: Is there an Environmental Factor?», Schizophr. Bull., 1998, 24 (4), pp. 529-535.

(32) Chaffin, M.; Kelleher, K.; Hollenberg, J., «Onset of Physical Abuse and Neglect: Psychiatric, Substance Abuse, and Social Risk Factors from Prospective Community Data», Child Abuse Negl., 1996, 20 (3), pp. 191-203.

(33) D’Amato, T.; SaOud, M., «Risk Factors and Schizophrenia», Encephale, 1996, 22 (3), pp. 3-7.

(34) David, A. S., y otros, «IQ and Risk for Schizophrenia: a Population-Based Cohort Study», Psychol. Med., 1997, 27 (6), pp. 1.311-1.323.

(35) LEIDERMAN, E. A., «Indicadores tempranos de la esquizofrenia: ¿Podemos predecir la enfermedad?», Vertex, 2004, 13 (53), pp. 213-219.

(36) Kagan, J.; ZenTnER, M., «Early Childhood Predictors of Adult Psychopathology», Harv. Rev. Psychiatry, 1996, 3 (6), pp. 341-0.

(37) Bertolote, J.; McGorry, P., «Early Intervention and Recovery for Young People with Early Psychosis: Consensus Statement,» British Journal of Psychiatry, 2005, 187 (48), pp. 116-119.

(38) Fòrum Salut Mental, Proposta de desenvolupament d'un model d'atenció als trastorns psicòtics incipients, «Document de treball», Barcelona, Fòrum de Salut Mental, 2006.

(39) International Early Psychosis Association, «Writing Group International Clinical Practice Guidelines for early Psychosis», British Journal of Psychiatry, 2005, 187 (48), pp. 120-124.

(40) LaluCAT, LL.; Tizón, J. L. (coords.), Programa d'atenció específica al trastorn psicòtic incipient del Pla Director de Salut Mental i Addiccions de la Generalitat de Catalunya, Barcelona, Generalitat de Catalunya, Servei Catalá de la Salut, 2006. 
ORIGINALES Y REVISIONES

(41) Marshall, M.; Lockwood, A., «Early Intervention for Psychosis» (Review), The Cochrane Library, 3, Londres, Wiley, 2003.

(42) MCGORRY, P. D.; JACKSON, H. J. (eds.), The Recognition and Management of early Psychosis. A Preventive Approach, Cambridge, Cambridge U.P., 1999.

(43) McGorry, P. D., «Royal Australian and New Zealand College of Psychiatrists Clinical Practice Guidelines for the Treatment of Schizophrenia and Related Disorders», Australian and New Zealand Journal, 2005, 39, pp. 1-30.

(44) NICE, Schizophrenia. Care Interventions in the Treatment and Management of Schizophrenia in Primary and Secondary Care, Clinical Guideline 1, Londres, NICE, 2002.

(45) Malla, A. K.; Norman, R. M. G.; Joober, PH., «First-Episode Psychosis, Early Intervention, and Outcome: Have we Learned?», Can J. Psychiatry, 2005, 50, pp. 881-91.

(46) Wно-Oмs, «Conclusions from Pre-conference "The Mental Health of Children and Adolescents"», organised by European Commission, WHO European Region and the Ministry of Health of Luxembourg, 2004, Ginebra, Wно-OMs, 2005.

(47) WhO-OMs, Health and Adolescent Mental Health Policies and Plans, WHO-OMs, Ginebra, 2005. (http://www.who.int/mental_health/policy/en/Child\%20\%20Ado\%20Mental\%20Health.pdf).

(48) Read, J.; Mosher, L.; Bentall, R. (eds.), Models of Madness: Psychological, Social and Biological Approaches to Schizophrenia, Londres, Brunner-Routledge, 2004 (traducción española, Modelos de Locura, Barcelona, Herder, 2006).

(49) National Institute of Mental Health, Genetics and Mental Disorders, Report of the National Institute of Mental Health's Genetics Workgroup, Rockville, NIMH, 1998.

(50) InSTITUTE OF MEDICINE, Reducing Risk for Mental Disorders: Frontiers for Preventive Intervention Resarc., Washington, The National Academy of Sciences, 2000.

(51) Isohanni, I., y otros, «School Performance as a Predictor of Psychiatric Hospitalization in Adult Life. A 28-year Follow-up in the Northern Finland 1966 Birth Cohort», Psychol. Med., 1998, 28 (4), pp. 967-4.

(52) JONES, P. B., y otros, «Schizophrenia as a Long-Term outcome of Pregnancy, Delivery, and Perinatal Complications: a 28-Year Follow-up of the 1966 North Finland General Population Birth Cohort», Am. J. Psychiatry, 1998, 155 (3), pp. 355-64.

(53) Olin, S. C.; Mednick, S. A., «Risk Factors of Psychosis: Identifying Vulnerable Populations Premorbidly», Schizophr. Bull., 1996, 22 (2), pp. 223-40.

(54) Olin, S. C., y otros, «School Teacher Ratings Predictive of Psichiatric Autcome 25 Years Later», Br. J. Psychiatry, sup. 1998, 172 (33), pp. 7-13.

(55) KonNECKE, R., y otros, «Main Risk Factors For Schizophrenia: Increased Familial Loading and Pre and Peri-natal Complications Antagonize the Protective Effect of Oestrogen in Women», Schizophr. Res., 2000, 44, pp. 81-3.

(56) United States Department of Health and Human Services, «Mental Health: A Report of the Surgeon General. Rockville, M. D, United States Department of Health and Human Services, Substance Abuse and Mental Health Services Administration», Centre for Mental Health Services, National Institutes of Health, National Institute of Mental Health, 1999.

(57) VAn Os, J., y otros, «Risk Factors Onset and Persistence of Psychosis», Soc. Psychiatry Psychiatr. Epidemiol., 1998, 33 (2), pp. 596-5.

(58) Who-Oms, WHO European Ministerial Conference on Mental Health (enero, 2005), «Facing the Challenges, Building solutions», WHO-OMS, Ginebra, 2005.

(59) Ellison, Z.; VAn Os, J.; MurraY, R., «Special Feature: Childhood Personality Characteristics of Schizophrenia: Manifestations of, or Risk Factors for, the Disorder?», J. Personality Disord., 1998, 12 (3), pp. 247-61. 
(60) ARTigue, J., y otros, «Una aproximación a la detección precoz de los trastornos mentales graves», Revista de Psicopatología del Niño y del Adolescente, 2004 (4), pp. 51-7.

(61) TIZÓN, J. L., Introducción: Prevención de los trastornos de la salud mental, en PAPPS y SEMFYC (eds.), Curso a distancia de prevención en atención primaria, Barcelona, SEMFYC y PAPPS, 1997, 1999, 2003, pp. 5-31.

(62) TIZÓN, J. L., «¿Se puede pensar todavía en una "sociogénesis" y en una "psicogénesis" de la psicosis?», Archivos de Psiquiatría, 2004, 67 (1), pp. 67-72.

(63) Guedeney, A.; Fermanian, J. A., «Validity and Reliability Study of Assessment and Screening for Sustained Withdrawal Reaction in Infancy: The Alarm Distress Baby Scale», Infant. Ment. Health J., 2001, 22 (5), pp. 559-575.

(64) Guedeney, A.; Charron, J.; Delour, M.; Fermanian, J., «L'évaluation du comportement de retrait relationnel du jeune enfant lors de l'examen pédiatrique par l'échelle d'Alarme Détresse Bébé (ADBB)», Psychiatr. Enfant., 2001, 44, pp. 211-231.

(65) ZabAla, I.; MANZANO, J., «Psicopatología durante la infancia en un grupo de adultos esquizofrénicos», Informaciones Psiquiátricas, 1993, 134 (4), pp. 381-387.

(66) MANZANO, J. (ed.), Las relaciones precoces entre padres e hijos y sus trastornos, Madrid, Necodisne, 2001.

(67) SAloKANGaS, R. K., «Living Situation Social Network and outcome in Schizophrenia: a Five-year Prospective Follow-Up Study», Acta Psychiatr. Scand., 1997, 96, 6, pp. 459-468.

(68) SuvisaARI, J. M., y otros, «Age at Onset and Outcome in Schizophrenia Are Related to the Degree of Familial Loanding», Br. J. Psychiatry, 1998, 173, pp. 494-0.

(69) Herrera, R., y otros, «Estudio epidemiológico en salud mental de la comarca del Baix Llobregat (Barcelona)», Informaciones Psiquiátricas, 1990, 120, pp. 111-30.

(70) VÁzquez-BARquero, J. L., y otros, «Sex Differences in Mental Illness: A Community Study of the Influence of Physical Health and Sociodemographic Factors», Soc. Psychiatry Epidemiol., 1992, 27, pp. 62-8.

(71) VÁzQueZ-BARQuero, J. L., y otros, «Sociodemographic and Clinical Variables as Predictors of the Diagnostic Characteristics of HealthGate Document», Acta Psychiatr. Scand., 1996, 94 (3), pp. 149-5.

(72) Harrow, M.; Jobe, T. H., «Longitudinal Studies of Outcome and Recovery in Schizophrenia and Early Intervention: Can They Make a Difference?», Can J. Psychiatry, 2005, 50 (14), pp. 1-4.

(73) Wно-Oмs, «Prevention of Mental Disorders: Effective Interventions and Policy Options: a Summary Report», Ginebra, World Health Organization, 2004. (www.who.int/mental_health/evidence/en/prevention_of_mental_disorders_sr.pdf).

(74) LARSEN, T. K., y otros, «Can Duration of Untreated Illness Be Reduced?», en BIRCHwOOD, M.; Fowler, K.; JACKSON, C. (eds.), Early Intervention in Psychosis: a Guide to Concepts, Evidence and Interventions, Chichester, Wiley, 2000, pp. 143-165.

(75) LARSEN, T. K., y otros, «Shortened Duration of Untreated First Episode of Psychosis: Changes in Patient Characteristics at Treatment», American Journal of Psychiatry, 2001, 158, pp. 1917-1919.

(76) Olsen, K. A.; Rosenbaum, B., «Prospective Investigations of the Prodromal State of Schizophrenia: Assessment Instruments», Acta Pscyhiat. Scan., 2006, 113, pp. 273-282.

(77) Smith, J.; SAhIERS, D., «Early Intervention in Psychosis Teams», 2006, en ISPSINT@yahoogroups.com. 
(78) FuChS, J.; SteinerT, T., «Patients with a First Episode of Schizophrenia Spectrum Psychosis and their Pathways to Psychiatric Hospital care in South Germany», Soc. Psychiatry Psychiatr. Epidemiol., 2004, 39, pp. 375-80.

(79) YunG, A., y otros, «Psychosis Prediction: 12-Month Follow Up of a High-Risk "Prodromal" Group», Schizophrenia Research, 2003, pp. 21-32.

(80) MeIssels, S. J.; Shonkoff, J. P. (comps.), Handbook of early Childhood Intervention, Cambridge, Press Sybdic of the University of Cambridge, 1990.

(81) JANÉ-LlOPIS, E., «La eficacia de la promoción de la salud mental y la prevención de los trastornos mentales», Revista de la AEN, 2004, 89, pp. 67-79.

(82) García-Ibáñez, J.; Tizón, J. L. (coords.), Grup de Treball, «Factor de Risc i Programes Preventius per a 0-12 anys», Pla Director de Salut Mental i Adiccions de la Generaltiat de Catalunya, Barcelona, Generalitat de Catalunya i Servei Catalá de la Salut, 2006.

(83) TizóN, J. L., «Psicopatología relacional de las Psicosis: Apuntes para la fundamentación del tratamiento psicológico de los trastornos psicóticos», Psicosomática y Psicoterapia, 2000, 20 (44), pp. 25-78.

(84) Tizón, J. L., «Epidemiología de la psicosis desde los servicios comunitarios de salud mental y de Atención Primaria de Salud», Archivos de Psiquiatría, 2004, 67 (2), pp. 139-144.

(85) Tizón, J. L., Propuesta de Programa de Atención Integral al Paciente Psicótico (PAIPP), Barcelona, Unitat de Salut Mental de Sant Martí-La Mina, Institut Catalá de la Salut, 1999 (polic.).

(86) Tizón, J. L., La investigación empírica sobre las psicoterapias: ¿Otra lente del «Hubble» defectuosa?, Vallejo, J. (dir.), Update de Psiquiatría 1997, Barcelona, Masson, 1997, pp. 67-99.

(87) Cullberg, J., y otros, «Integrating Intensive Psychosocial and Low-Dose Neuroleptic Treatment: a Three-Year Follow-Up», en MarTindale, B. (ed.), Psychosis: Psychological Approaches and their Effectiveness, Londres, Gaskel-ISPS, 2000, pp. 200-210.

(88) JACKSON, M., Weathering the Storms: Psychotherapy for Psychosis, Londres, Karnac Books, 2001.

(89) MARI, J. J.; STREINER, D., «Family Intervention for Schizophrenia», en AdAMS, C. E., y otros (eds.), Schizophrenia Module of the Cochrane Database of Systematic Reviews, Oxford, Update Software, 1998.

(90) Peterson, L., y otros, A Randomised Multicentre Trial of Integrated Versus Standard Treatment for Patients with a First Episode of Psychotic Illness, BMJ, 2005, 331, pp. 602 .

(91) Gleeson, J. F. M.; McGorry, P. D. (eds.), Psychological Interventions in early Psychosis. A Treatment Handbook, Chichester, Wiley, 2004, pp. 1-22.

(92) TIzÓN, J. L.; SAN-José, J.; NADAL, D., Protocolos y programas elementales para la atención primaria a la salud mental, Barcelona, Herder, 2000.

(93) TızóN, J. L., «Terapias combinadas en la esquizofrenia: ¿Agregamos, mezclamos y confundimos o bien de-construimos y combinamos?», Revista de la AEN, 2004, 14 (90), pp. 97-131.

(94) Oliver, J., y otros, «Measuring the Quality of Life of Severely Ill People Using the Lancashire Quality of Life Profile», Social Psychiatry and Psychiatric Epidemiology, 1997, 32, pp. 76-3.

(95) HäFNER, H., y otros, «Onset and Early Course of Schizophrenia», en HäFNER, H.; GatTAZ, W. F. (eds.), Search for Causes of Schizophrenia, III, Berlín, Springer, 1995, pp. 43-66.

(96) HäFNER, H., y otros, «IRAOS: An Instrument for the Assessment of the Onset and early Course of Schizophrenia», Schizophrenia Research, 1992, 6, pp. 209-23.

(97) Perkins, D., y otros, «Characterizing and Dating the Onset of Symptoms in Psychotic Illnes: the Symptom Onset in Schizophrenia (SOS) Inventory», Schizophr. Res., 2000, 44, pp. 1-10. 
(98) Yung, A. R., y otros, Monitoring and Care of Young People at Incipient Risk of Psychosis, Schizophr. Bull., 1996, 22 (2), pp. 283-03.

(99) Yung, A. R.; McGorry, P. D., «The Prodromal Phase of First-episode Psychosis», Schizophrenia Bulletin, 1996, 22 (2), pp. 353-370.

(100) Yung, A. R., y otros, «Prediction of Psychosis. A Step towards Indicated Prevention of Schizophrenia», Br. J. Psychiatry, supl. 1998, 172 (33), pp. 14-0.

(101) Yung, A. R, y otros, «Risk Factors for Psychosis in an Ultra High-risk Group: Psychopathology and Clinical Features», Schizophrenia Research, 2004, 67, pp. 131-142.

(102) Yung, A., y otros, «Mapping the Onset of Psychosis: the Comprehensive Assessment of a at-Risk Mental States», Journal of Psychiatry, Australia y Nueva Zelanda, 2005, pp. 964-971.

(103) Amigó, E., y otros, Programa de Seguiment del Nen Sa: Protocol de Salut Mental Infantil i Juvenil, Barcelona, Direcció General de Salut Pública. Departament de Sanitat i Seguretat Social de la Generalitat de Catalunya, 1999 (Programa de detección precoz de la Generalitat de Catalunya, en cuya redacción participó el investigador principal).

(104) Achenbach, T. M., Manual for the Child Behavior Checklist/2-3 and 1992 Profile, Burlington, University of Vermont, Department of Psychiatry, 1992.

(105) Bourgeors, M. L., Les schizophrénies, París, PUF, 1999.

(106) CABAsÉs, J. M.; VILlABí, J. R.; AIBAR, C. (eds.), Informe SESPAS 2000: Invertir para la salud. Prioridades en Salud Pública, Valencia, Generalitat Valenciana- Escuela Valenciana de Estudios para la Salud, 2002.

(107) Saldivia Bohórquez, S.; Torres GonzÁlez, F.; Cabases Hita, J. M.; Psicost Group, «Estimation of Mental Health care Cost Units for Patients with Schizophrenia», Actas Esp. Psiquiatr., 2005, 33 (5), pp. 280-5.

Agradecimientos: A Bent Rosenbaum por su orientación bibliográfica; a Anna Oriol por su colaboración en la organización de la bibliografía del artículo.

* J. L. Tizón es director del Equipo de Prevención en Salud Mental-Equipo de Atención Precoz a los Pacientes con Psicosis (EAPPP) del Institut Catalá de la Salut en Barcelona.

Correspondencia: Jorge L. Tizón, Prevenció en Salut Mental-EAPPP. Tel. 934360004 - Fax. 934355 303; jtizon.pbcn@ics.scs.es.

** Fecha de recepción: 10-XI-2006 (aceptado el 20-XI-2007). 